\title{
Natural Resources Research: Reviewers in 2019
}

\author{
John Carranza ${ }^{1,2}$
}

Improving and maintaining the standard of papers in this journal depend on the selfless time and effort of the many scientists who review manuscripts. We acknowledge and thank the following individuals for reviewing manuscripts submitted to our journal during 2019; some of them have reviewed more than one manuscript.

Abdel-Fattah, M. I.

Abdideh, M. $(2 \times)$

Abdullah, W. H. B. $(2 \times)$

Abedi, M. $(4 \times)$

Abzalov, M.

Agterberg, F. $(2 \times)$

Ahmadi, M.

Ahmed, M. A. $(2 \times)$

Akanji, L. T.

Al-Abadi, A.

Aldhaheri, M.

Ali, M.

Alipour, A.

Alizamir, M.

Al-Mudhafar, W. J.

Al-Saedi, H. $(2 \times)$

Al-Shuhail, A. A.

Alvarez, R.

Amanipoor, $\mathrm{H}$.

Amici, A.

Amiri, M.

Amnieh, H. B. $(2 \times)$

Ampomah, W.

Anderson, S. T.

Andrews-Speed, P.

Armaghani, D. J. $(9 \times)$

\footnotetext{
${ }^{1}$ Durban, South Africa.

${ }^{2}$ To whom correspondence should be addressed; e-mail: ejmcarranza@gmail.com
}

We apologize in case any names have been omitted inadvertently.
Arumaikkani, G. S.

Arzani, A.

Asghari, O. $(2 \times)$

Ashtari, A.

Aslan, A.

Assareh, M.

Audigane, $\mathrm{P}$.

Avramidis, $\mathrm{P}$.

Awaleh, M. O.

Aydin, G.

Aydin, M.

Azevedo, L.

Azhdarpour, A.

Azizi, A.

Baba, A.

Bagheri, R. $(2 \times)$

Bakhshandeh Amnieh, H. $(2 \times)$

Bakhshi, E.

Bakhtavar, E.

Bandyopadhyay, S. $(2 \times)$

Baran, P.

Bartzas, G.

Barzegar, R.

Bassani, M.

Beg, M. K.

Benavente, D.

Benndorf, J. $(2 \times)$

Bérubé, C. L.

Bhakar, P.

Bhattacharya, S.

Bielik, M.

Bildirici, M. E.

Bilim, F.

Biondić, R.

Biswas, A. $(8 \times)$

Boisvert, J. $(2 \times)$

Bonakdari, $\mathrm{H}$.

Bonham-Carter, G. F. $(3 \times)$

Bortoluzzi, E. C. 
Bowell, R.

Brito, A. B.

Buccianti, A.

Bucha, M.

Budhu, M.

Bui, D. T. $(6 \times)$

Bülbül, A.

Bunger, A.

Bürger, C. M.

Burnham, A. K.

Cao, W.

Cao, Y.

Carasco, C.

Careddu, N.

Carranza, E. J. M. $(2 \times)$

Castro, L.

Cengiz, O.

Cerqueira Koppe, V. $(2 \times)$

Chai, J.

Chalavadi, G.

Challa, V.

Chanda, E.

Chandrasekar, N.

Chang, C.

Chang, L.

Chatterjee, R. N. $(3 \times)$

Chatterjee, S. $(2 \times)$

Chen, B.

Chen, G. $(2 \times)$

Chen, K.

Chen, L. $(2 \times)$

Chen, S.

Chen, W. $(2 \times)$

Chen, X.

Chen, Y. $(6 \times)$

Chen, Z.

Cheng, Y.

Cherubini, C.

Chiu, Y. $(3 \times)$

Choi, Y.

Christmann, P.

Chu, T.

Ciceri, D.

Çırmık, A.

Coburn, T. $(2 \times)$

Cohen, D. R. $(5 \times)$

Coto, B.

Coyan, J. A.

Cracknell, M. J.

Culver, T. B.

Dabrowska, D. $(3 \times)$

Dai, S. $(2 \times)$
Dash, P. K.

Datta, B.

Davarpanah, A. $(3 \times)$

David, C.

De Paz, J. M.

Deb, D.

Dehghani, H. $(2 \times)$

Dehghanpour, $\mathrm{H}$.

Dhar, A.

Diab, Z.

Dixon, D. S.

Dokou, Z. A.

Dominy, $S$.

Dong, S.

Dou, L.

Drerup, S. A.

Dutta, S.

Egermann, P.

Ekinci, Y. L.

El-Bastawesy, M. A.

Eldursi, K.

Elhag, M.

Elliott, E. G.

Elsayed, Y.

Elsharkawy, A.

Elshkaki, A.

Emery, X. $(4 \times)$

Engle, $\mathrm{M}$.

Er, C.

Eremin, M.O.

Ersoy, A.

Esterhuizen, G. S.

Ewida, H. F.

Fabbri, A. G. $(2 \times)$

Fan, B. $(2 \times)$

Fan, G.

Fan, L.

Fan, Y. $(2 \times)$

Fang, R.

Farquharson, C. G.

Farrell, N. J. C.

Farzin, S.

Feltrin, L.

Feng, G. $(2 \times)$

Fiket, Ž.

Filzmoser, P.

Fink, R.

Ford, A. $(3 \times)$

Foroud, T.

Förster, A.

Fouedjio, F. $(3 \times)$

Fraser, S. 
Freulon, X. $(2 \times)$

$\mathrm{Fu}, \mathrm{X}$.

Galhardi, J. A.

Gamache, M.

Gamage, R.

Gangopadhyay, P.

Gao, W.

Genc, B.

Geng, J.

George, N. J. $(2 \times)$

Ghaderpoori, M.

Ghasemi, E. $(4 \times)$

Gholami, R.

Ghorbani, H.

Ghorbanzadeh, O.

Gil-Alana, L. A.

Giri, A. K.

Gomo, M.

Goncalves, M. $(2 \times)$

Gong, Q.

Gonzalez, A.

Gözgör, G.

Graedel, T.

Green, S.

Guellala, R. $(4 \times)$

Gumiel, P.

Gunning, A.

Guo, C.

Guo, L.

Guo, P. $(2 \times)$

Gupta, S. D.

Gutzmer, J.

Hajihassani, M. $(2 \times)$

Hakim, S.

Hakimi, M. H.

Hall, G.

Harris, J. R. $(5 \times)$

Hartmann, A.

Hasanipanah, M. $(8 \times)$

Hastie, W. W. $(2 \times)$

$\mathrm{He}, \mathrm{H}$.

Hemmati-Sarapardeh, A.

Heydari, A.

Hilson, G.

Hingston, E. $(2 \times)$

Höök, M. $(2 \times)$

Hower, J.

Hron, K. $(2 \times)$

$\mathrm{Hu}, \mathrm{Q}$.

Huang, B.

Hutahaean, J.

Iqbal, M. A.
Jaafar, M. Z.

Jaafari, A.

Jackish, R.

Jafrasteh, B.

Jalali, M. $(7 \times)$

Jensen, J.

Jeong, C.

Jiang, F. $(2 \times)$

Jiang, L.

Jiang, $\mathrm{T}$.

Jin, L.

Jing, Y.

Kaffine, D.

Kaiser, M. $(2 \times)$

Kalantar, B.

Kanoua, W.

Karakus, D. $(2 \times)$

Karayigit, A. I.

Kareem, B.

Karimi, M.

Kataka, M. O. $(2 \times)$

Keppert, M.

Khalid, P.

Khamehchi, E.

Khandelwal, M. $(4 \times)$

Khodaparast, M. $(2 \times)$

Khormali, A.

Khosravi, M.

Kim, H.

King, B.

Kishore, N.

Knight, J.

Koike, K.

Konaté, A. A.

Koopialipoor, M. $(4 \times)$

Kosakowski, P.

Kovalevs'ka, I.

Kreuzer, O. $(3 \times)$

Krishnaraj, S. M.

Kristjanpoller, W.

Krooß, B. M.

Kuma, J. S. $(3 \times)$

Kumar, S.

Kumral, M. $(9 \times)$

Kunal, P.

Kuosmanen, T.

Kursun, I.

Kutitani, T.

Lai, J. $(2 \times)$

Lalehzari, R.

Lashin, A.

Latif, Z. 
Laugier, F.

Lavrov, A.

Leahy, P.

Lee, J.-Y.

Lee, $\mathrm{K}$.

Lee, S.-G. $(2 \times)$

Leonzio, G.

Li, C.

Li, E.

Li, H. $(2 \times)$

Li, J.

Li, M. $(5 \times)$

Li, N.

Li, Q.

Li, R. $(2 \times)$

Li, S. $(2 \times)$

Li, W.

Li, X. $(3 \times)$

Lima, H.

Lishchuk, V.

Liu, B.

Liu, F.

Liu, G.

Liu, J.

Liu, P. $(2 \times)$

Liu, S.

Liu, T. $(2 \times)$

Liu, X.

Liu, X. S.

Liu, Y. $(14 \times)$

Loganathan, N.

Loupasakis, C. J.

Lu, J.

Luo, Y.

Lyu, C.

Maanijou, M.

Madani, N. $(3 \times)$

Malykh, O. E.

Manning, D. $(3 \times)$

Manzione, $\mathrm{R}$.

Mao, X.

Marrugo-Negrete, J. L.

Martos, Y.

McKinley, J. M.

McLellan, B. C. $(2 \times)$

Mehrabi, H.

Meng, M.

Michaelides, S. $(2 \times)$

Minnitt, R. $(3 \times)$

Miroshnichenko, D. V.

Mirzaei-Paiaman, A.

Mishra, A. K.
Mogaji, K. A.

Mohamed, M.

Mohapatra, S. S.

Mohialdeen, I. $(2 \times)$

Mokaya, X.

Molina, E.

Monjezi, M. $(4 \times)$

Moussa, T. M.

Mueller, U.

Mukerji, T.

Mungatana, E.

Murphy, T.

Nabawy, B. $(2 \times)$

Nader, B.

Nadiri, A. A.

Nag, S. K.

Naghibi, S. A. $(8 \times)$

Najibi, A. R.

Nandy, S.

Nchor, D.

Neuman, S. P.

Nguyen, H. $(15 \times)$

Nguyen, P.

Nhamo, L.

$\mathrm{Ni}, \mathrm{G}$.

Niri, M. E.

Noorollahi, Y. $(3 \times)$

Nwaila, G. $(2 \times)$

Nykänen, V.

Obracaj, D.

Ojha, K. $(2 \times)$

Olea, R. $(2 \times)$

Omeragić, D.

Omotehinse, A. O.

Önder, M.

Onifade, M.

Ordonez-Calderon, J. C.

Ortiz, J. M. $(2 \times)$

Osterman, G.

Othman, A. A.

Ovinis, M.

Oyelami, C. A.

Ozfirat, M. K.

Padhy, P. K.

Pagnoncelli, B.

Pal, S.

Pan, G. $(2 \times)$

Pan, J. $(2 \times)$

Pan, Z.

Parnachev, S. V.

Partington, G. A.

Pathak, S. 
Patiño Douce, A. E.

Paton, D. A.

Pawlowsky-Glahn, V.

Peng, S.

Peng, W.

Pham, T. D.

Porwal, A.

Prasad, R.

Prol-Ledesma, R. M. $(2 \times)$

$\mathrm{Pu}, \mathrm{H}$.

Qin, L.

Rabbani, A. R.

Radwan, A.

Rahaman, S. A.

Rahmani, O.

Rahmati, O. $(9 \times)$

Raina, A. K.

Rajabi, A. M. $(2 \times)$

Rajendran, S. $(2 \times)$

Ramalingam, $\mathrm{P}$.

Rani, S.

Ranjbar, $\mathrm{H}$.

Rasmussen, T. M.

Rathnaweera, T.

Ray, S. K.

Raziperchikolaee, S.

Reading, A. M.

Rekapalli, R.

Riccioli, F.

Rivoirard, J.

Rizvi, R. H.

Romanak, K. D.

Rondon, O.

Roumpos, C.

Rudakov, D. V.

Rui, Z.

Saad Saoud, L.

Saboori, B.

Sadeghi, M. $(2 \times)$

Sadeghnejad, S. $(3 \times)$

Sáez, A. E.

Sahebraoji Nimaje, D.

Sahoo, S.

Sahoo, U. C.

Sahu, S. K.

Saibi, H.

Saikia, B.

Saikia, N.

Sakala, E. $(3 \times)$

Sako, A.

Samal, A. R.

Samal, A. R. $(2 \times)$
Sánchez, L. E.

Sang, S.

Sanguinito, S.

Sanmiquel, L.

Sanuade, O. A.

Sappa, G.

Schuenemeyer, J. H.

Sen, S.

Sen, Z.

Şener, M. F.

Sengupta, M.

Shalaby, M. R. $(2 \times)$

Shan, L.

Shan, X.

Shang, D. $(3 \times)$

Shang, $X$.

Shankar, U.

Sharifi, M.

Shen, C. B. $(2 \times)$

Shen, L.

Sheng, M.

Shi, J. Q.

Shi, L.

Shi, P.

Shi, X. $(3 \times)$

Shields, D.

Shin, $\mathrm{H}$.

Shkuratnik, V. L. $(3 \times)$

Shnorhokian, S.

Shoji, T.

Shokrollahi, A.

Shu, L.

Si, G.

Silversides, K. L.

Singer, D. A.

Singh, A.

Singh, A. K. $(2 \times)$

Singh, O.

Singh, S.

Singha, D. K.

Sípos, P. I.

Smith, L.

Solarin, S. A.

Soleimani, B.

Soleimani, M. $(2 \times)$

Solomon, N.

Somorowska, U.

Song, $\mathrm{M}$.

Song, W.

Song, $\mathrm{X}$.

Sorjonen-Ward, P.

Sousa, L. 
Suh, J.

Sui, L.

Sun, F. $(7 \times)$

Sun, T. $(3 \times)$

Sun, Z.

Suwartadi, E.

Swami, D.

Syed, T. H.

Szafranek-Nakonieczna, A.

Talavera, O.

Tan, M.

Tang, S. B.

Tangestani, M. H.

Tariq, Z.

Tarrio, D.

Tatar, A.

Tavchandjian, O.

Tedeschi, A.

Tercan, E.

Thomas, M. D.

Tian, Y.

Tilton, J. E.

Todorović, B.

Tóth, Á.

Tran, Q.

Tziritis, E.

Vangeepuram, R.

Vargas, C.

Varouchakis, E. A.

Vasić, M. V.

Vass, C. R.

Verba, C.

Verma, A. K.

Viccaro, M. $(4 \times)$

Vishal, V. $(2 \times)$

Vural, A.

Wagner, N.

Wang, A.

Wang, E.

Wang, G.

Wang, J.

Wang, K.

Wang, L. $(2 \times)$

Wang, $M$.

Wang, Q.

Wang, S. $(3 \times)$

Wang, W. $(3 \times)$

Wang, $\mathrm{Z}$.

Wei, B. $(2 \times)$

Wei, J.

Woche, S. K.

Woldai, T.
Wood, D. $(4 \times)$

Wood, S.

Wu, D. $(4 \times)$

$\mathrm{Wu}, \mathrm{K}$.

$\mathrm{Wu}, \mathrm{W}$.

Xiao, F. $(2 \times)$

Xiao, K.

Xiao, L.

Xiao, X.

Xie, S.

Xion, J.

$\mathrm{Xu}$, C. $(2 \times)$

$\mathrm{Xu}, \mathrm{G}$.

$\mathrm{Xu}, \mathrm{H}$.

$\mathrm{Xu}$, J. $(2 \times)$

$\mathrm{Xu}, \mathrm{L}$.

$\mathrm{Xu}, \mathrm{S}$.

$\mathrm{Xu}, \mathrm{Y} .(2 \times)$

Xue, D. J.

Yamamoto, J. K.

Yan, F. $(2 \times)$

Yan, $\mathrm{H}$.

Yang, D.

Yang, J. $(2 \times)$

Yaseen, Z. M.

Yasukawa, K.

Yaya, O. S.

Ye, H.

Ye, Z.

Yin, $Y$.

Yoneda, $\mathrm{T}$.

Yousefi, M. (14×)

$\mathrm{Yu}, \mathrm{B}$.

$\mathrm{Yu}, \mathrm{M}$.

$\mathrm{Yu}, \mathrm{Y}$.

Yuan, F. $(3 \times)$

Yuan, Y.

Yunpei, L.

Zahid, A.

Zendehboudi, S.

Zhang, B.

Zhang, C. $(2 \times)$

Zhang, D. $(5 \times)$

Zhang, K. $(2 \times)$

Zhang, N.

Zhang, T.

Zhang, X. $(3 \times)$

Zhang, Y. $(2 \times)$

Zhang, Z. $(3 \times)$

Zhao, F.

Zhao, G.

Zhao, H. 
Zhao, Y.

Zhe, C. $(8 \times)$

Zheng, $\mathrm{P}$.

Zhou, F.

Zhou, J. $(2 \times)$

Zhou, T.

Zhu, L.

Ziemba, P.

Ziggah, Y. Y.

Ziyong, $\mathrm{Z}$.

Zolotukhin, A. B.

Zou, H.

Zou, Q.

Zou, Y.
Zouaghi, T.

Zoveidavianpoor, M. $(2 \times)$

Zuo, R. (3x)

Zuzolo, D.

In 2019 in total 869 manuscripts were submitted for consideration to Natural Resources Research. After a careful and timely peer-review process 151 papers were accepted for publication in 2019, and still ca. 50 manuscripts submitted in 2019 are under review.

John Carranza

Editor-in-Chief 